\title{
Evaporation of an anisotropic nanoplasma
}

\author{
Camila Bacellar ${ }^{1,2}$, Adam S. Chatterley ${ }^{1}$, Florian Lackner $^{1}$, C.D. Pemmaraju ${ }^{1}$, Rico M. P. \\ Tanyag $^{3}$, Charles Bernando ${ }^{4}$, Deepak Verma ${ }^{3}$, Sean O'Connell ${ }^{3}$, Maximilian Bucher ${ }^{5}$, \\ Kenneth R. Ferguson ${ }^{6}$, Tais Gorkhover ${ }^{6,7}$, Ryan N. Coffee ${ }^{6}$, Giacomo Coslovich ${ }^{6}$, \\ Dipanwita Ray $^{6}$, Timur Osipov ${ }^{6}$, Daniel M. Neumark ${ }^{1,2}$, Christoph Bostedt ${ }^{5,8^{*}}$, Andrey F. \\ Vilesov $^{3,4^{*}}$, and Oliver Gessner ${ }^{1 *}$

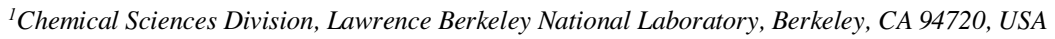 \\ ${ }^{2}$ Department of Chemistry, University of California Berkeley, Berkeley, CA 94720, USA \\ ${ }^{3}$ Department of Chemistry, University of Southern California, Los Angeles, CA 90089, USA \\ ${ }^{4}$ Department of Physics and Astronomy, University of Southern California, Los Angeles, CA 90089, \\ USA \\ ${ }^{5}$ Argonne National Laboratory, 9700 South Cass Avenue B109, Lemont, IL 60439, USA. \\ ${ }^{6}$ Linac Coherent Light Source, SLAC National Accelerator Laboratory, Menlo Park, CA 94025, USA \\ ${ }^{7}$ Institute of Optics and Atomic Physics, Technical University of Berlin, 10623 Berlin, Germany \\ ${ }^{8}$ Department of Physics and Astronomy, Northwestern University, Evanston, IL 60208, USA
}

\begin{abstract}
Intense laser induced plasma dynamics in sub-micron scale helium droplets are monitored by femtosecond time-resolved X-ray coherent diffractive imaging. Anisotropic surface softening and strongly anisotropic shrinking of the plasma core are observed.
\end{abstract}

\section{Introduction}

Laser induced plasmas generated in sub-micron sized atomic clusters have long been used as finite, replenishing model systems to study the complex correlated electronic and nuclear dynamics of highly ionized systems. A simplified overview of the plasma evolution is generally described by a three-step model [1]. First, the atoms in the cluster are strong-field ionized during the leading edge of an intense laser pulse. This is followed by the formation of a nanoplasma due electrons being trapped in the collective Coulomb potential of the positive nuclei. Finally, the cluster expands via hydrodynamic expansion, Coulomb explosion, or a combination of both processes depending on the average mass and charge state of the cluster. A variety of spectroscopy and imaging experiments as well as simulations have confirmed fundamental aspects of this picture but also provided evidence for more complex dynamics, such as surface melting effects $[2,3]$ and anisotropic ion and electron kinetic energy emissions [4-6], rather than uniform cluster expansion. A complete, real-time picture of the cluster dynamics upon nanoplasma formation is still outstanding. Here, we monitor the entire evolution of nanoplasmas form by exposing helium droplets to a strong

*Corresponding authors: cbostedt@anl.gov,vilesov@usc.edu,ogessner@lbl.gov 
NIR laser field from the onset of anisotropic surface softening to the strongly anisotropic droplet disintegration.

\section{Experimental setup and results}

Helium droplets with average diameters of $\approx 600 \mathrm{~nm}$ are generated by expansion of high pressures (20 bar) of He gas through a cold $(4.7 \mathrm{~K}) 5 \mu \mathrm{m}$ wide nozzle. In the interaction region, the droplet beam is intercepted by a femtosecond NIR laser beam (wavelength $\lambda=800 \mathrm{~nm}$, pulse width $\tau \approx 40 \mathrm{fs}$, focal size $2 \omega \approx 75 \mu \mathrm{m}$ ) at intensities of $\approx 4 \times 10^{15} \mathrm{~W} / \mathrm{cm}^{2}$, leading to strong-field ionization of $\mathrm{He}$ atoms and subsequent nanoplasma formation and cluster disintegration. The dynamics are monitored by recording single-particle X-ray diffraction patterns, each generated by the interaction of a single LCLS X-ray pulse $(\lambda=2.07 \mathrm{~nm}, \tau \approx 100 \mathrm{fs}, 2 \omega \approx 5-10 \mu \mathrm{m})$ with a single ionized droplet at NIR-pump/X-rayprobe delays between -20 and 100 ps. A single-shot NIR/X-ray cross-correlator is used to measure the relative pump-probe delay with a precision of $\approx 24 \mathrm{fs}$ (FWHM). Small angle (37$140 \mathrm{mrad}) \mathrm{X}$-ray scattering patterns are detected using a two-panel pnCCD X-ray camera positioned perpendicular to the X-ray/NIR beams $360 \mathrm{~mm}$ downstream of the interaction region. Signals from scattered NIR light are suppressed by leaving large $(28 \mathrm{~mm})$ vertical
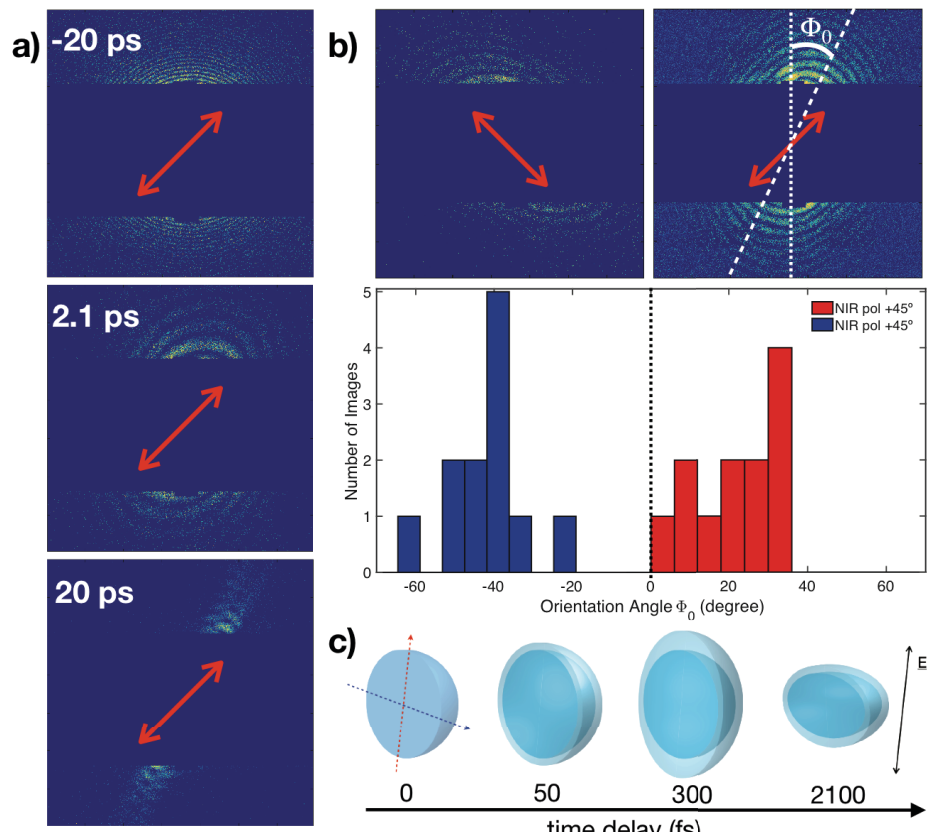

c)

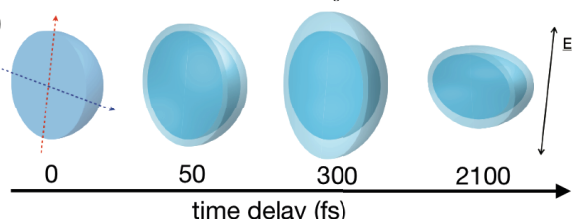

Fig. 1. a) Representative X-ray diffraction patterns for three pump-probe time delays $\Delta t$ at a constant NIR polarization (red arrows). b) Representative images (top) and orientation distribution (bottom) of elliptical diffraction patterns at a NIR-pump / X-ray-probe delay of $2.1 \mathrm{ps}$. Patterns are recorded for NIR polarization angles of $\pm 45^{\circ}$ as indicated by the red double arrows. c) Artistic representations of droplets at various stages of the nanoplasma evolution show relative core sizes and aspect ratios to scale. The extent and anisotropy of the surface regions has been exaggerated for improved clarity. 
gaps between the detector panels and the laser beam axis and by mounting $220 \mathrm{~nm}$ thick Al foils to the front of the panels. The polarization angle of the linearly polarized NIR light is controlled using a $\lambda / 2$ waveplate.

Representative single-shot diffraction patterns corresponding to a variety of time delays between the NIR pump pulse and the X-ray probe pulse are shown in Fig. 1a, in which a substantial change in the shape and ring spacing of the images as a function of time delay and, in particular, a strong correlation between the laser polarization axis and the pattern orientations are readily apparent. The CDI images can be fit to simulated X-ray diffraction patterns of biaxial spheroids from which we obtain the transient droplet sizes, surface widths and orientation angles. The droplets consistently exhibit shorter core radii (Fig. 1b) and larger surface widths parallel to the NIR polarization, up to their complete disintegration for $\Delta \mathrm{t}>20$ ps.

Anisotropic surface softening is observed within tens of femtoseconds after exposure to the NIR pulse and continues over $\approx 300 \mathrm{fs}$, after which the anisotropic surface profile stabilizes with $a \approx 30 \%$ larger extension along the laser polarization axis compared to the perpendicular direction (not shown). The saturation of the surface width is contrasted by an increase in anisotropic material loss that is twice as pronounced along the laser polarization axis, resulting in significantly distorted plasma shapes with aspect ratios of $\approx 1.5$ and beyond. Fig.1c shows an artistic representation of the nanoplasma evolution.

The results are interpreted using a numerical simulation of the ejection dynamics of atoms from the plasma within an anisotropic nanoplasma evaporation model, supported by angleresolved ion kinetic energy distributions that are recorded in coincidence with the X-ray diffraction patterns. The simulation captures key aspects of the observed plasma shape evolution very well, in particular, the saturation of surface softening within hundreds of femtoseconds and the continued anisotropic melting of the core proceeding on picosecond timescales. This study provides a connection between these newly observed dynamics in large nanoplasmas approaching micron scales and previous studies [1-3] on much smaller systems.

This work was supported by the U.S. Department of Energy, Office of Science, Office of Basic Energy Sciences, Chemical Sciences, Geosciences and Biosciences Division, through Contract No. DE-AC0205CH11231.

\section{References}

1. T. Ditmire, T. Donnell, A. M. Rubenchik, R. W. Falcone and M. D. Perry, "Interaction of intense laser pulses with atomic clusters.” Phys. Rev. A 53, 3379-3402 (1996).

2. C. Peltz, C. Varin, T. Brabec, and T. Fennel, "Time-resolved x-ray imaging of anisotropic nanoplasma expansion.” Phys. Rev. Lett. 113,133401 (2014).

3. T. Gorkhover, et al. "Femtosecond and nanometre visualization of structural dynamics in superheated nanoparticles." Nat. Photonics 10, 93-97 (2016).

4. V. Kumarappan, M. Krishnamurthy, and D. Mathur, "Asymmetric high-energy ion emission from argon clusters in intense laser fields.” Phys. Rev. Lett. 87, 085005, (2001).

5. M. Hirokane, S. Shimizu, M. Hashida, S. Okada, S. Okihara, F. Sato, T. Iida, and S. Sakabe, "Energy distributions of ions emitted from argon clusters Coulomb-exploded by intense femtosecond laser pulses" Phys. Rev. A 69, 063201

6. E. Skopalova, Y. C. El-Taha, A. Zaïr, M. Hohenberger, E. Springate, J. W. G. Tisch, R. A. Smith, and J. P. Marangos, "Pulse-length dependence of the anisotropy of laser-driven cluster explosions: transition to the impulsive regime for pulses approaching the fewcycle limit.” Phys. Rev. Lett. 104, 203401, (2010). 